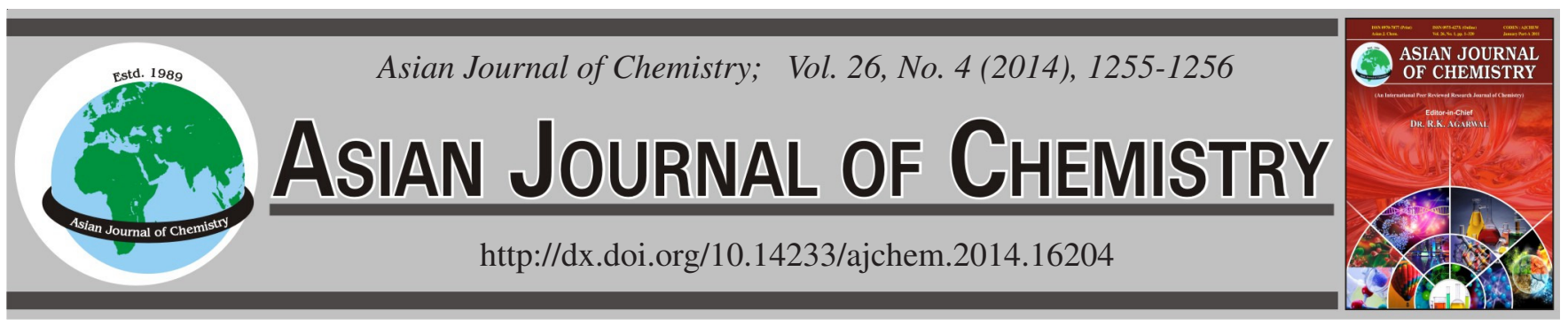

NOTE

\title{
Effect of Stretching on Properties of Poly(L-lactic acid)/ 1,2,3-Benzotriazole Composites: A Comparative Study
}

\author{
YAN-HuA CAI ${ }^{*}$ and YAN-HuA ZHANG
}

Chongqing Key Laboratory of Environmental Materials \& Remediation Technologies, Chongqing University of Arts and Sciences, Yongchuan, Chongqing 402160, P.R. China

*Corresponding author: E-mail: caiyh345@gmail.com

\begin{abstract}
Poly(L-lactic acid) (PLLA)/1,2,3-benzotriazole (BTA) composites were fabricated by melt blending and hot-press forming process. The melting behavior of stretched PLLA/2 \% BTA composities was investigated by differential scanning calorimetry. The results showed that stretching could improve the crystallization ability of poly(L-lactic acid) and stretching made crystallization peak of poly(L-lactic acid) become sharper and the high-temperature melting peak become more obvious. Addition of 1,2,3-benzotriazole also could improve the crystallization of poly(L-lactic acid). However, the crystallization peak of stretched PLLA/2 \% BTA samples became weak. Melting behavior of stretched PLLA/BTA samples was similar to that of PLLA/BTA, the low-temperature melting peak shifted to higher temperature with increasing of crystallization temperature. However, the high-temperature melting peak almost did not move.
\end{abstract}

Keywords: Poly(L-lactic acid); 1,2,3-Benzotriazole, Melting behaviour, Composites.

With reduction of oil resource, biodegradable polymers have attracted more and more attention due to their biodegradability. Poly(L-lactic acid) (PLLA) is one of the most important biodegradable polymers and have been used in more and more field such as materials ${ }^{1}$, medicine ${ }^{2}$, chemistry ${ }^{3}$, etc. However, PLLA has some disadvantages to limit its application ${ }^{4}$. In order to improve the application field of PLLA, the various influence factors of PLLA and its composites were investigated to meet the application requirements. Stretching is phenomenon occurred during application of polymer and stretching will significantly affect the properties of polymer, but there exists little relevant research. Thus, in this paper, effect of stretching on the properties of PLLA/2 \% 1,2,3-benzotriazole composites was investigated and the results may help us understand the performance of PLLA/BTA composites.

Poly(L-lactic acid) (2002D) was purchased from Nature Works LLC, USA. 1,2,3-Benzotriazole was procured from Chengdu Kelong Chemical Reagents Company, China.

Preparation of PLLA/BTA sample: Blending of PLLA and BTA was performed on a counter-rotating mixer and the preparation process of PLLA/2 \% BTA composites has described in our previous paper 5 .

The melting behaviour of PLLA and PLLA/2 \% BTA was measured by DSC Q2000 (TA Instruments-Waters LLC, USA). The temperature and heat flow at different condition were calibrated using an indium standard.
Fig. 1 shows the melting behaviour of neat PLLA and stretched PLLA at heating rate of $10^{\circ} \mathrm{C} / \mathrm{min}$. It is clear that there exists crystallization peak and double-melting peaks during heating. As to double-melting peaks, the low-temperature melting peak is attributed to the primary crystallites formed at crystallization temperature and high-temperature melting peak reflect the relatively prefect lamella stacks results from recrystallization during the heating $\operatorname{scan}^{6}$. Compared to neat PLLA, stretching makes crystallization peak of PLLA become sharper and the high-temperature melting peak becomes more obvious. Moreover, the ratio of area between high-temperature melting peak and low-temperature melting peak becomes large. These results indicated that stretching improves the crystallization ability of PLLA and the degree of crystallization increases.

Fig. 2 shows the melting behaviour of PLLA with $2 \%$ BTA at heating rate of $10{ }^{\circ} \mathrm{C} / \mathrm{min}$. As seen in Fig. 2, compared to the neat PLLA, the crystallization peak of PLLA becomes sharper after addition of BTA, which indicates that BTA improves the crystallization of PLLA. However, the crystallization peak of stretched PLLA/2 \% BTA samples becomes weak, meantime, the intensity of melting peak also becomes weak. The possible reason is that strectching makes segment of PLLA be more regular, however, on the other hand, addition of BTA makes the regular arrangement of PLLA become more difficulty and addition of BTA also makes segment of PLLA move more difficulty. 


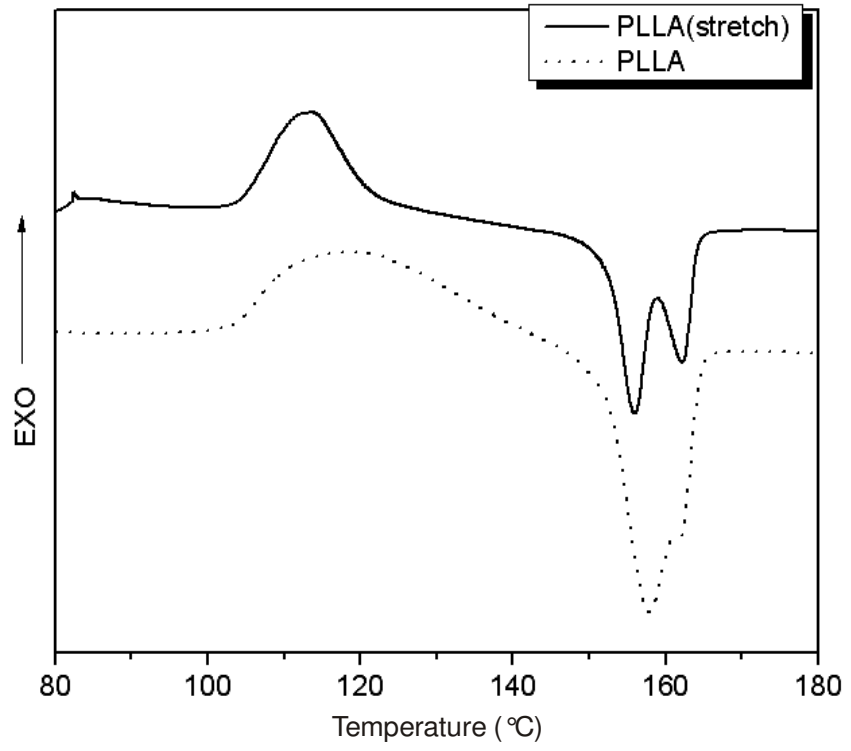

Fig. 1. Melting behaviour of neat PLLA and stretched PLLA at heating rate of $10^{\circ} \mathrm{C} / \mathrm{min}$

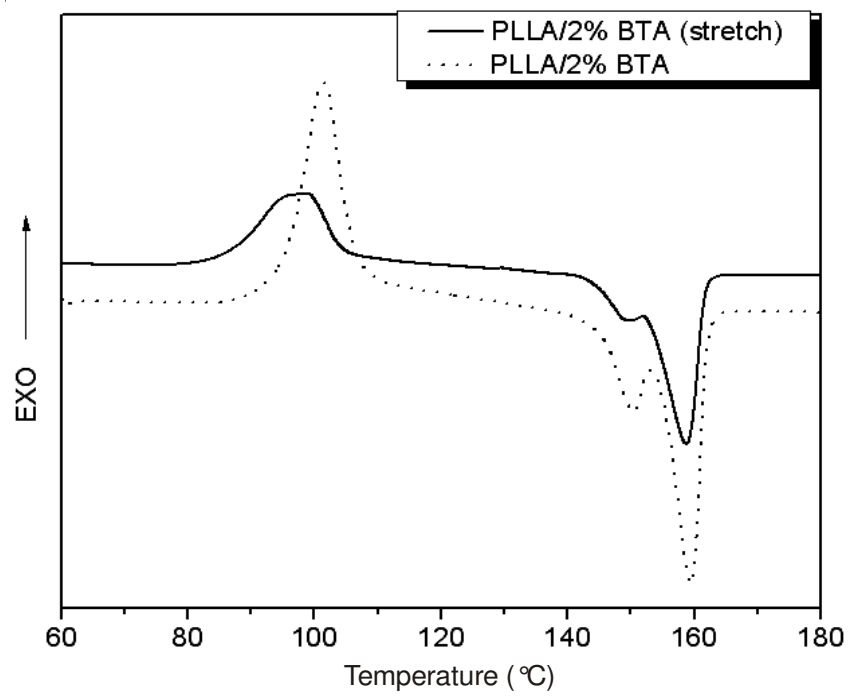

Fig. 2. Melting behaviour of PLLA/2 \% BTA sample at heating rate of $10{ }^{\circ} \mathrm{C} / \mathrm{min}$

Melting behaviour of PLLA/BTA samples after crystallization: Fig. 3 shows the melting behaviour of PLLA/BTA after crystallization for $1 \mathrm{~h}$ at different crystallization temperature. As shown in Fig. 3, the low-temperature melting peak shift to higher temperature with increasing of crystallization temperature. However, the high-temperature melting peak almost do not move, resulting from more prefect crystal with increasing of crystallization temperature, what is more, the ratio of area between low-temperature and high-temperature melting peak increases with increasing of crystallization

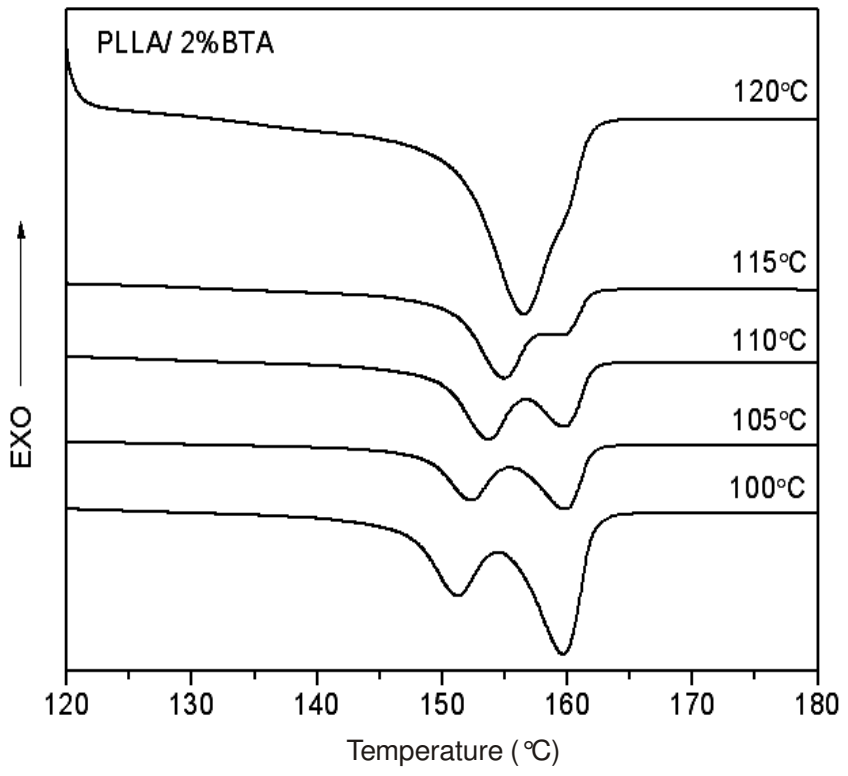

Fig. 3 Melting behaviour of PLLA/BTA sample after crystallization

temperature. The possible reason is that the moving ability of molecular chain segment increases at same crystallization time with increasing of crystallization temperature and these exists more prefect crystal. Moreover, it is clear that the melting behaviour of stretched PLLA/BTA samples is similar to that of PLLA/BTA sample.

\section{ACKNOWLEDGEMENTS}

This work was supported by China Postdoctoral Science Foundation (project number 2013M531937), Natural Science Foundation Project of CQ CSTC (project number cstc2012jjA50001), Postdoctoral Science Foundation Project of Chongqing (Project No. XM20120035), Scientific and Technological Research Program of Chongqing Municipal Education Commission (project number KJ131202) and Chongqing University of Arts and Sciences (Project number R2012CH10 and 2012PYXM04).

\section{REFERENCES}

1. J.Z. Liang, D.R. Duan, C.Y. Tang, C.P. Tsui and D.Z. Chen, J. Macromol. Sci. Part B Phys., 52, 964 (2013).

2. S.P. Zhan, C. Chen, Q.C. Zhao, W.J. Wang and Z.J. Liu, Ind. Eng. Chem. Res., 52, 2852 (2013).

3. L. Peponi, I. Navarro-Baena, A. Sonseca, E. Gimenez, A. MarcosFernandez and J.M. Kenny, Eur. Polym. J., 49, 893 (2013).

4. Y. Cai, S. Yan, Y. Fan, Z. Yu, X. Chen and J. Yin, Iran. Polym. J., 21, 435 (2012)

5. Y. Cai, S. Yan, J. Yin, Y. Fan and X. Chen, J. Appl. Polym. Sci., 121, 1408 (2011).

6. Y.-H. Cai, E-J. Chem., 9, 1569 (2012). 